\title{
Fibrolipoma of the Rib: A Case Report and Review of the Literature
}

\author{
Shantanu Pande ${ }^{1}$, Zaeba Nayeem², Bhagat Singh Lali ${ }^{1}$, Zachariah Chowdhury ${ }^{1}$, Deepak Kumar Singh ${ }^{1}$ \\ ${ }^{1}$ Homi Bhabha Cancer Hospital and Mahamana Pandit Madan Mohan Malviya Cancer Center, Sunder Bagiya, BHU campus, \\ Varanasi, Uttar Pradesh, India, 221005; ${ }^{2}$ Tata Memorial Hospital, Dr E Borges Road, Parel, Mumbai, India.
}

\section{Corresponding Author:}

Dr Shantanu S. Pande

Email: quantnucmed@gmail.com

This is an Open Access article distributed under the terms of the Creative Commons Attribution License (creativecommons.org/ licenses/by/3.0).

Received

Accepted

Published
March 22, 2021

July 22, 2021

August 5, 2021

\begin{abstract}
Background: Fibrolipoma is a rare histological variant of lipoma and although fibrolipomas of the oral cavity have been reported, only a few cases of fibrolipoma of the thoracic region have been published. Case Report: A 49-year-old man presented with pain and a lump in the left side chest. After initial evaluation, CT followed by PET/CT were acquired. The scan findings were suggestive of a benign lipomatous tumor of the left $4^{\text {th }}$ rib; however, malignant transformation could not be ruled out. Hence, excision of the rib lesion with mesh repair and flap reconstruction was performed. Post-operative histopathology was suggestive of a fibrolipoma with absence of atypia or mitosis. Radiological and pathological findings and management of the case are discussed below. Conclusion: Follow-up of such rare tumors are essential to detect recurrence as knowledge about the behavior of these tumors is limited.
\end{abstract}

Keywords: Lipoma, Male, Pain, Ribs, Surgical Mesh, Thorax.

\section{Introduction}

Fibrolipoma is a rare soft tissue tumor with distinct pathology. Histologically it is a variant of lipoma, which is the most common benign soft tissue tumor [1]. A lipoma is composed of mature adipocytes with varying proportions of mesodermal components. Liposarcoma is a malignant variant of lipoma. Fibrolipomas are rare variants of lipomas with a high component of fibrous tissue. They develop from lipoblastomatosis, the maturation of which causes fat globules to become separated by fibrous strands [2-4]. Fibrolipomas of the oral cavity have been reported [5]; however, fibrolipoma of the rib is extremely rare, and to the best of our knowledge, only four cases of fibrolipomas in the thoracic region have been published. We report the presentation and management of one such case at our institute.

\section{Case Report}

A 49-year-old man presented with pain and a lump in the left side of his chest for a duration of
5-6 months. There was associated dyspepsia, but no known co-morbidities. Clinical examinations revealed a solitary, spherical, firm, left chest wall swelling of approximately $8 \mathrm{~cm}$ diameter. The skin over the swelling showed no significant changes and the rest of the medical examination was noncontributory.

Chest X-ray revealed a well-defined radiopaque lesion involving the left $4^{\text {th }}$ rib [Fig.1]. Computed tomography (CT) of the thorax confirmed these findings [Fig.2]. In addition, the lesion showed macroscopic fat and septa with an average Hounsfield unit (HU) of -45 . In a bone window, the lesion showed sclerotic margins. There was no obvious enhancing soft tissue or erosion of bone. No calcified matrix was noted. Benign-looking nodules were seen in the right lung. The remainder of the scan was unremarkable. Radiological differential diagnosis consisted of intraosseous lipoma and lipomatous tumors (angiolipoma and myelolipoma), hemangioma, a solid variant of aneurysmal bone cyst, and bone 


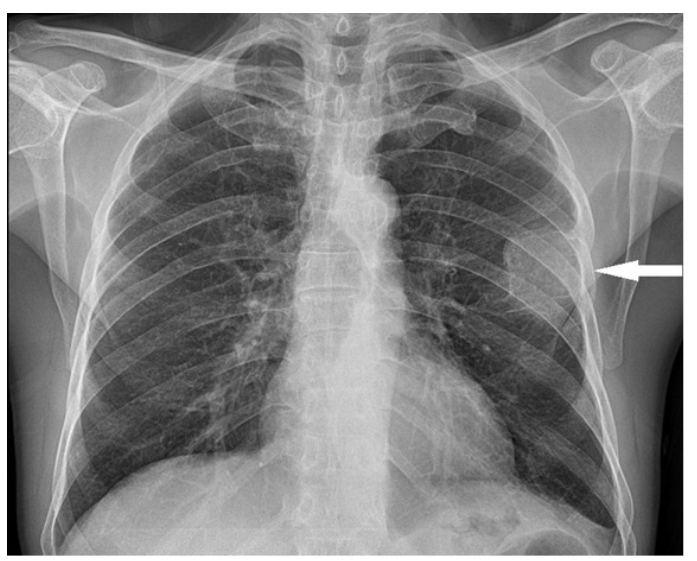

Fig.1: Chest radiograph (PA view) showed a well-defined radiopaque lesion involving the anterior left $4^{\text {th }}$ rib (arrow).

infarct. Rare differentials included liposarcoma and metastases from fat-containing lesions. 18F-Fluorodeoxyglucose positron emission tomography/computed tomography (18F-FDG PET/CT) showed no evidence of metabolic activity in the rib lesion [Fig.3]. There was no evidence of active disease elsewhere and no possible site of a primary malignancy could be located. These findings suggested that the mass was a benign lipomatous tumor. The patient underwent excision of the left $4^{\text {th }}$ rib lesion with mesh repair and a pectoralis myo-cutaneous muscle flap reconstruction. The gross examination of the excised rib showed an outer bulge on the medial side and cut surface showed an intraosseous, yellow colored solid lesion/tumor with size of $5 \times 4.5 \times$ $2.6 \mathrm{~cm}$ [Fig.4]. The histopathological examination of the lesion showed lobules of mature adipocytes separated by fibrovascular septae. There were intermixed areas of fibroblastic proliferation with spindle shaped to plump ovoid cells arranged in short fascicles. The surrounding stroma showed thick and wavy collagen fibres with occasional spicules of bone. There was absence of cytologic atypia and increased mitosis. The bone marrow was uninvolved and showed normal hematopoiesis. There were no post-operative complications and the patient was discharged on the $7^{\text {th }}$ post-operative day. At 2 months follow-up the patient was doing well and had no complaints.
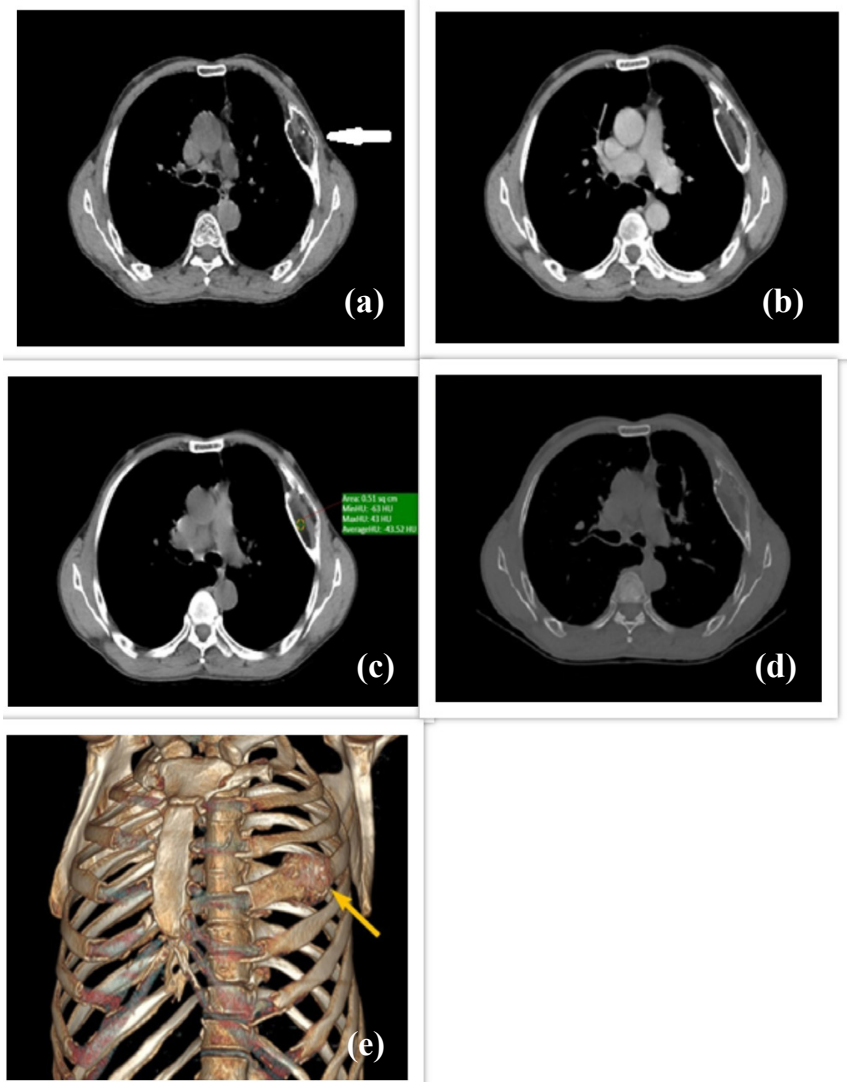

Fig.2: (a): Axial plain CT chest image showed a welldefined expansile lytic lesion involving the left anterior $4^{\text {th }}$ rib (arrow); (b): Post-contrast image showed no obvious enhancing component; (c): The lesion showed macroscopic fat within with average $H U$ of -45; (d): In bone window, the lesion showed sclerotic margins; (e): $3 D$ volume rendered image showed lytic lesion In the left $4^{\text {th }} \mathrm{rib}$.

\section{Discussion}

Fibrolipoma is most frequently seen as a variant of oral lipoma. Fibrolipomas of the thoracic region are extremely rare. To the best of our knowledge, this is only the fifth case reported in the literature $[6,7]$, in addition few cases of intraosseous lipoma of rib have been reported. Lipomas and their variants have a characteristic radiological picture. In plain radiographs, they show well-defined radiolucency [8]. CT images exhibit fat attenuation of approximately $-100 \mathrm{HU}$. In the later stages, calcification due to fat necrosis well-defined sclerotic borders can be seen. Additional imaging in the form of magnetic resonance imaging (MRI) 
and PET/CT scans is required to characterize the tumor and differentiate it from liposarcoma. MRI shows signal intensity that is isointense with that of subcutaneous tissue [9]. Based on margins, signal homogeneity (non-fatty areas), thick septations or nodules in MRI scans, and uptake in 18F-FDG PET/CT, benign and malignant tumors can be differentiated $[9,10]$. However, there can be overlap $\neg$ ping metabolic activity of benign and malignant lipomatous tumors on 18F-FDG PET/ CT [11]. The pre-operative diagnosis of any soft tissue sarcoma includes radiology followed by histological examination in the form of needle biopsy, or in very large tumors an incisional biopsy may be justified.

In this case, the tumor showed fatty attenuation with no metabolic activity in PET/CT; hence, it was considered a benign lipomatous tumor rather than a malignancy. However, completely excluding the malignant nature of a lesion can be difficult. Biopsy can be performed for histological evaluation, but it can be difficult to differentiate various histological types of lipomatous tumors, particularly when limited tissue is available for assessment. Hence, in our case, based on the patient's complaints and limits on tissue sampling, we decided to resect the lesion.

Lipomatous tumors must be differentiated from liposarcomas. Kamata et al. [6] reported a similar case of intrathoracic fibrolipoma; they argued that frozen section analysis is not an ideal method to differentiate between benign lipomatous tumors and liposarcomas. Histologically, lipomas are benign tumors of adipose tissue. Histological variants of lipoma include angiolipoma, chondroid lipoma, fibrolipoma, myelolipoma, myolipoma, myxolipoma, ossifying lipoma, sclerotic lipoma, and adenolipoma. These are named based on the components admixed with mature adipocytes [12]. The differential diagnosis of fibrolipoma includes pleomorphic lipoma and atypical lipomatous tumor sclerosing variant. Fibrolipoma does not exhibit pleomorphism or cytological atypia in adipocytic
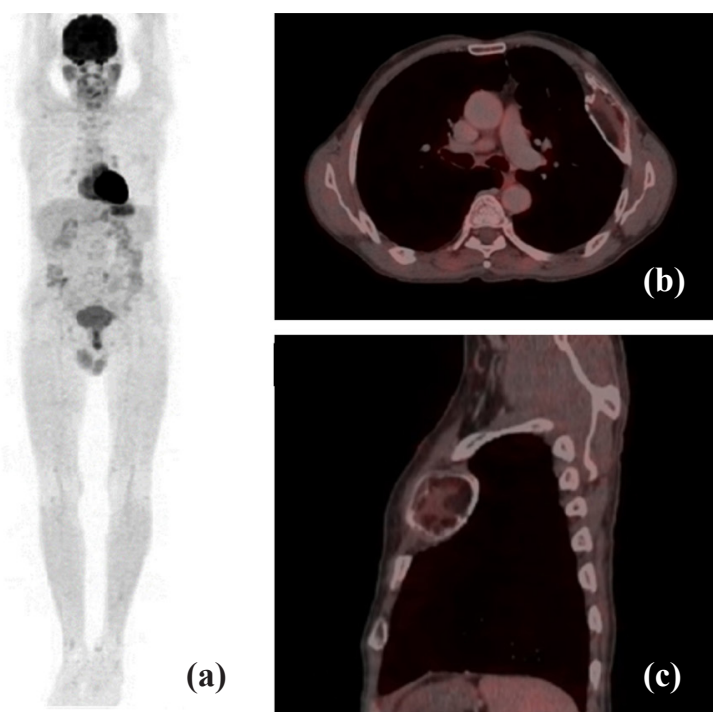

(a)

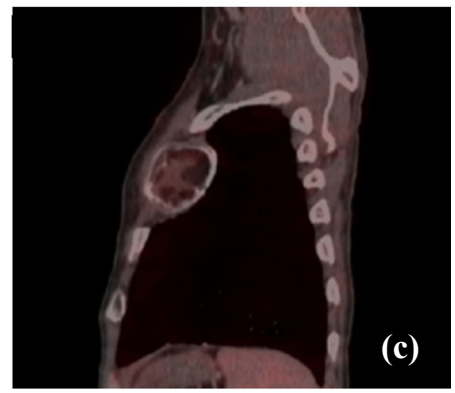

Fig.3: (a): Maximum intensity projection image showed no evidence of abnormal FDG uptake; (b,c): Axial and sagittal fused PET/CT images showed no FDG uptake in rib lesion.
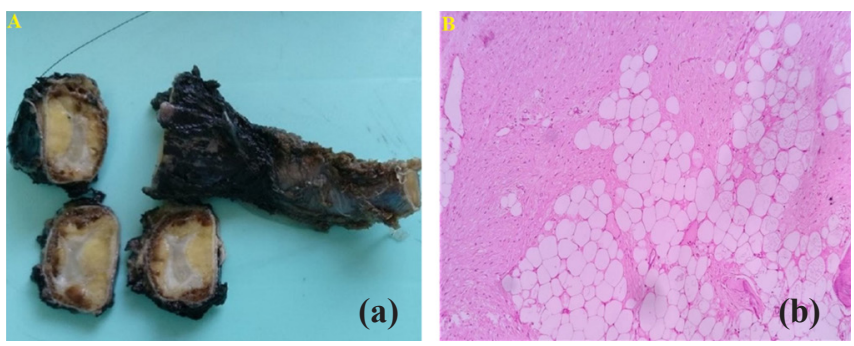

Fig.4: (a): Gross cut surface of the rib showed an intraosseous, yellow coloured solid lesion/tumor within the bony trabeculae; (b): Histology of the tumor showing mature adipocytes with fibroblastic proliferation and occasional bony spicules.

or fibroblastic cells. However, pleomorphic lipoma contains pleomorphic adipocytes, occasionally shows pseudo-lipoblasts, and typically has 'ropy' collagen. Cytological atypia is always present in atypical lipomatous tumors, together with a floret morphology of the stromal cells [13].

Rare cases of malignant transformation in intraosseous lipoma have been reported [14], though such a transformation has not been reported until recently in a fibrolipoma [15]. Treatment is usually surgical excision with negative margins, which offers a complete cure. Most of these tumors 
are asymptomatic; however, as in our case, patients may have symptoms of local nerve involvement or compression of the lung parenchyma. Surgery is usually performed to improve functional outcomes and post-operative radiotherapy is not indicated, according to a review of the current literature. However, careful observation and follow-up are essential to detect the possibility of recurrence early in the course of disease, particularly because this is a rare entity and our knowledge about the behavior of these tumors is limited [16].

\section{Conclusion}

The fibrolipoma of rib is extremely rare. The lesion can be asymptomatic and discovered incidentally but presentation can be with pain. Malignancy cannot be ruled out completely on imaging findings and excision remains as modality of treatment with close follow-up to rule out recurrence.

Contributors: SP, BSL: manuscript writing, patient management; ZN: manuscript editing; ZC, DKS: critical inputs into the manuscript. AL will act as a study guarantor. All authors approved the final version of this manuscript and are responsible for all aspects of this study.

Funding: None; Competing interests: None stated.

\section{References}

1. Murphey MD, Carroll JF, Flemming DJ, Pope TL, Gannon FH, Kransdorf MJ. From the archives of the AFIP: benign musculoskeletal lipomatous lesions. Radiogr Rev Publ Radiol Soc N Am Inc. 2004;24:14331466.

2. Dagistan E, Barut AY, Karagöz Y, Fener N, Canan A, Kizıldağ B. The radiological appearance of a giant intrathoracic fibrolipoma. Tuberk Toraks. 2016;64:87-90.

3. Hsu JS, Kang WY, Liu GC, Kao EL, Chuang MT, Chou SH. Giant fibrolipoma in the mediastinum: an unusual case. Ann Thorac Surg. 2005;80:e10-12.
4. Gaerte SC, Meyer CA, Winer-Muram HT, Tarver RD, Conces DJ Jr. Fat containing lesions of the chest. Radiographics. 2002;22:S61-78.

5. Khubchandani M, Thosar N, Bahadure R, Baliga MS, Gaikwad R. Fibrolipoma of buccal mucosa. Contemporary Clinical Dentistry. 2012;Supplement 1;e112-114.

6. Kamata S. Ishida I, Suzuki Y, Yamada T, Yaegeshi $\mathrm{H}$, Oura H. Intrathoracic fibrolipoma resected using complete thoracoscopic surgery: a case report. Journal of Cardiothoracic Surgery. 2018;13:112.

7. Peacock MJ, Craddock DR, Allen PW. Pleural fibrolipoma: report of a case. Aust NZ J Surg. 1974; 44:117-119.

8. Atilla E, Gundogdu C, Atila T, Nurettin K, Erzurum. Intraosseous lipoma of the rib. J Thorac Cardiovasc Surg. 2005;130:1468-1469.

9. Brisson M, Kashima T, Delaney D, Tirabosco R, Clarke A, Cro S, et al. MRI characteristics of lipoma and atypical lipomatous tumor/well-differentiated liposarcoma: retrospective comparison with histology and MDM2 gene amplification. Skeletal Radiol. 2013;42:635-647.

10. Suzuki R, Watanabe H, Yanagawa T, Sato J, Shinozaki $\mathrm{T}$, Suzuki H, et al. PET evaluation of fatty tumors in the extremity: possibility of using the standardized uptake value (SUV) to differentiate benign tumors from liposarcoma. Ann Nucl Med. 2005; 19:661-670.

11. Baffour F, Wenger D, Broski M. 18 F-FDG PET/CT imaging features of lipomatous tumors. Am J Nucl Med Mol Imaging. 2020;10:74-82.

12. Fletcher CDM, Bridge JA, Hogendoorn PCW, Mertens F. Adipocytic tumours. In: WHO Classification of Tumours of Soft Tissue and Bone. $4^{\text {th }}$ ed. Lyon: International Agency for Research on Cancer; 2013. pp. 33-43.

13. Goldblum JR, Weiss SW, Folpe AL. Enzinger and Weiss's Soft Tissue Tumors, $6^{\text {th }}$ edition. 2014. Elsevier: Philadelphia.

14. Milgram JW. Malignant transformation in bone lipomas. Skeletal Radiol. 1990;19:347-352.

15. Kim YT, Kim WS, Park YL, et al. A case of fibrolipoma. Korean J Dermatol. 2003;41:939-941.

16. Jesberg N. Fibrolipoma of the pyriform sinuses: Thirtyseven year follow-up. Laryngoscope. 1982;92:11571159. 